\title{
BMJ Open Perception of modern radiotherapy learning: study protocol for a mixed- methods analysis of trainees and trainers at a UK cancer centre
}

\author{
Gerard Walls (D) , ${ }^{1,2}$ James J McAleer, ${ }^{2,3}$ Gerard G Hanna ${ }^{1,4}$
}

To cite: Walls G, McAleer JJ, Hanna GG. Perception of modern radiotherapy learning: study protocol for a mixed-methods analysis of trainees and trainers at a UK cancer centre. BMJ Open 2020;10:e037171. doi:10.1136/ bmjopen-2020-037171

- Prepublication history and additional material for this paper are available online. To view these files, please visit the journal online (http://dx.doi. org/10.1136/bmjopen-2020037171).

Received 22 January 2020 Revised 23 March 2020 Accepted 28 April 2020

Check for updates

(c) Author(s) (or their employer(s)) 2020. Re-use permitted under CC BY-NC. No commercial re-use. See rights and permissions. Published by BMJ.

${ }^{1}$ Centre for Cancer Research \& Cell Biology, Queen's University Belfast, Belfast, N. Ireland ${ }^{2}$ Cancer Centre, Belfast City Hospital, Belfast Health \& Social Care Trust, Belfast, N. Ireland

${ }^{3}$ Centre for Medical Education, Queen's University Belfast,

Belfast, UK

${ }^{4}$ Sir Peter MacCallum

Department of Oncology,

The University of Melbourne,

Melbourne, Victoria, Australia

Correspondence to

Dr Gerard Walls;

g.walls@qub.ac.uk

\section{ABSTRACT}

Introduction Radiotherapy technology and postgraduate medical training have both evolved significantly over the last 20 years. Clinical Oncology is a recognised craft specialty where the apprenticeship model of clinical training is applicable. The challenges of learning radiotherapy in the modern radiotherapy department workplace have not been comprehensively described and no optimal method has been identified.

Methods and analysis Five Clinical Oncology trainers and five Clinical Oncology trainees at a regional cancer centre will be invited to undertake a semistructured interview regarding their personal accounts of learning radiotherapy. Both trainees and consultants will be treated as equal co-investors in the process of radiotherapy learning, with the common shared aim of passing radiotherapy skills from trainers to trainees. Interviews will last up to $40 \mathrm{~min}$. After transcription, an interpretative phenomenological analysis will be performed. All trainees and trainers at the same centre $(n=34)$ will then be invited to complete the same purpose-built questionnaire. Four trainers and three trainees have piloted the questionnaire, and input was sought from the national leads of the biennial UK Clinical Oncology training survey. Significance testing will be performed on predefined questions and thematic analysis on white space questions.

Ethics and dissemination Medical education research is evolving in Clinical Oncology and Radiation Oncology but there are few studies comprehensively assessing this from the viewpoint of trainees and trainers. Pending the success of the proposed study, the approach detailed represents a novel method that could be used to identify the strengths and weaknesses of radiotherapy training in other centres and settings. Ethical and governance approvals have been granted by the University Research Ethics Committee and the Integrated Research Application System, respectively. This study has been funded by Friends of the Cancer Centre.

\section{INTRODUCTION}

Clinical oncologists care for patients with cancer by overseeing the delivery of their radiotherapy (RT) and systemic anticancer treatments (SACT) such as chemotherapy, endocrine therapy, targeted agents and immunotherapy. Trainees spend at least 5 years

\section{Strengths and limitations of this study}

- This is both the first 'mixed-methods' study within the area of radiotherapy training and the first study involving both trainers and trainees in the UK; therefore, the protocol for this approach merits replication and/or adaptation in other UK centres in the future.

- The questionnaire design involved several national figures in radiotherapy medical education, and as the Centre at the focus of the proposed study is regarded as contemporary, therefore, all investigators believe that recommendations from the study will be widely applicable.

- This is the first study focusing solely on radiotherapy in the Clinical Oncology model of training and thus, this study's results will provide feedback specifically to those responsible for radiotherapy teaching at local, regional and national levels.

- There is a risk of the lead researcher's unilateral perspective as a trainee influencing the results despite mindfulness for reflexivity.

under supervision before being accredited to practice independently. The training follows an apprenticeship model and is centred around day-to-day delivery of care, theoretical preparation courses, departmental group teaching and personal study. ${ }^{1}$ The practical nature of RT means that developing clinical competencies relies on experience in the workplace, and Clinical Oncology has been described as a craft specialty, ${ }^{23}$ with trainees and trainers assuming an apprenticeshiplike relationship. ${ }^{45}$ The last two decades have brought greatly improved RT outcomes for many patient groups through improved understanding of radiobiology and technological advances in computing and engineering. ${ }^{6}$ In preparing for ultimately taking up consultant posts, trainees must be familiar with the scientific principles of RT, for example, physics and radiobiology, the modern treatment techniques, and also the more conventional techniques. ${ }^{1}$ 
In 2005, the Modernising Medical Careers' initiative reformed postgraduate medical training, standardising the pathways to a career as a consultant in Clinical Oncology. ${ }^{7}$ Around the same time, enforcement of the European Working Time Directive affected working patterns and rota structures for trainees. ${ }^{8}$ The expansion of the Clinical Oncology consultant workforce also began to influence medical team structure. ${ }^{9}$ As a result of such adjustments on the background of a multifactorial national shortage of junior doctors, ${ }^{10}$ it is common for Clinical Oncology trainees to provide middle-grade cover for inpatients of several consultants simultaneously and attend multiple systemic therapy clinics each week, thereby regularly missing RT learning opportunities. ${ }^{3}$ This is reflected in a recent study that found new consultants feel under-prepared for independent RT practice when they take up their first post. ${ }^{11}$

Although supervised and systematic practice in hospitals facilitates the acquisition of competences, knowledge and skills, doctors are ultimately responsible for their own learning as members of training programmes. ${ }^{12}$ Educational agreements between trainees and their trainers require bilateral engagement and proactivity. ${ }^{13}$ In the last decade, documentation of learning has become increasingly important in the UK with the introduction of the ePortfolio system, ${ }^{14}$ which has served to formalise educational supervision ${ }^{15}$ and assessments. ${ }^{16} 17$

All UK doctors are also charged with the responsibility of teaching under the principles of Good Medical Practice as laid out by the General Medical Council. ${ }^{18}$ Professional revalidation procedures stipulate that active participation in education programmes must be demonstrated at 5-yearly appraisals. ${ }^{19} 20$ The Royal College of Radiologists (RCR) recognises the need for workplace-based training in its incorporation of dedicated teaching time in job planning recommendations for consultants ${ }^{21}$ and in its vision for the evolution of the specialty. ${ }^{22}$

The effects of the evolution in cancer outcomes and progress in RT techniques in recent decades have been studied by few educationalists in the UK, or further afield.$^{23}$ The natural focus on clinical research in this small hospital discipline partly accounts for this. The limited recent research available on training in general in Clinical Oncology suggests that the transformations in the RT landscape have had an impact on training. ${ }^{3} 611$ 24-28 This proposed research is a dedicated study of RT training among Clinical Oncology specialty trainees and consultants at a regional cancer centre to elicit their views on the current strengths and weaknesses of modern RT learning.

\section{METHODS AND ANALYSIS}

\section{Aims}

1. To qualify the strengths and weaknesses of RT learning at a regional cancer centre by assessing the views of trainees and trainers using semistructured interviews.

2. To quantify the strengths and weaknesses using a questionnaire of both trainers and trainees.
3. To identify differences between trainer and trainee perceptions of modern RT learning.

\section{Study design}

This is a mixed-methods research project with two phases of investigation. Phase I will consist of semistructured interviews with five trainers and five trainees. Phase II will comprise a questionnaire survey of all 36 trainers and trainees. For this study, all Clinical Oncology consultants are regarded as 'trainers' and all specialty trainees are regarded as 'trainees'.

Interpretive phenomenological analysis (IPA) will be performed on the qualitative data generated in phase I. Descriptive statistics will be applied to the quantitative data generated in phase II, and thematic analysis will be employed for phase II qualitative information. Phase I will establish the strengths and weaknesses of modern RT learning from a sample of Clinical Oncology doctors at Cancer Centre Belfast City Hospital (BCH) and phase II will generate numerical data on the entire population of Clinical Oncology doctors. The results from both phases will be considered in a pooled manner and also by trainer/trainee status to assess for practically significant differences between the experience and opinion of trainers compared with trainees. The original study proposal was developed iteratively following review by faculty members of Centre for Medical Education (CME) at Queen's University Belfast (QUB) and presentation at a CME Research Fellow Seminar.

\section{Methods: phase I}

Semistructured interviews allow the researcher to start with a planned open question and subsequently discuss unplanned threads of discourse depending on the interviewee's responses. ${ }^{29}$ The aim is to elicit details about RT learning settings from the perspectives of the trainee and the trainer, and how the training arrangements interact with the elements of RT practice perceived as easy or difficult to learn. Participants will be given the opportunity to describe their experiences, state their positive and negative opinions on modern RT learning and what they would change to improve it. Trainer and trainees will answer the same stem questions (figure 1) except for one question which applies only to trainers (indicated). The order in which the stems are asked will depend on the course of each individual dialogue, in keeping with the principles of semistructured interview. The number of stems introduced will depend on the duration of the interviewee's previous responses, as the lead investigator will aim to keep interview duration to approximately $40 \mathrm{~min}$ in total.

Describe your personal experience of training in radiotherapy.

What are the differences between training then and now (trainers only)

- Describe the better and poorer components of your own RL.

- Describe what you perceived as easy and difficult to learn regarding radiotherapy.

- Could trainees do more to enhance their radiotherapy learning currently?

- Could trainers do more to enhance radiotherapy learning currently?

Do you recognise any particular value of trainers and trainees 'contouring' together?

- What do you think of workplace-based assessments in relation to radiotherapy learning?

Figure 1 Stem interview questions. 


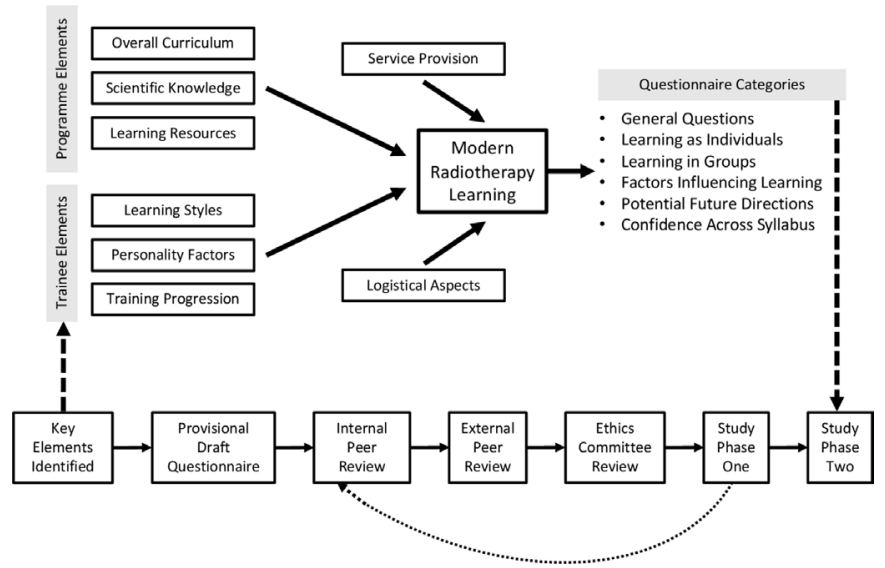

Figure 2 Outline of framework for questionnaire design and development.

At the completion of the semistructured interview, the participants' involvement will be concluded.

IPA will enable the lead investigator to elicit information about the lived experience of trainers and trainees, in part by 'bracketing' of his own exposure to RT learning to date ${ }^{30-32}$ Bracketing refers to the placement of ones' own judgements and perceptions aside, and approaching the presented information factually and neutrally, aiming to unpack the underlying detail logically. Advantages of this analysis include depth of output, catalysed by the lead investigator's informed contextual position of currently being in a Clinical Oncology training post. The lead investigator has been trained in the scientific rationale, practical requirements and conventional regulations of conducting semistructured interviews and conducting IPA during his successful completion of the Methods in Educational Enquiry module undertaken at QUB, as part of the Masters in Clinical Education. The research team will endeavour to apply the principles laid out in the COnsolidated criteria for REporting Qualitative research recommendations. ${ }^{33}$

\section{Methods: phase II}

Phase II data collection will be in the form of a questionnaire 4 weeks after completion of phase I (online supplementary appendices 1 and 2). The questionnaire was initially developed once key concepts to capture were agreed by the core research team (figure 2). The preliminary draft was iteratively developed using a combination of resources: Clinical Oncology curriculum, the Oncology Registrar's Forum (ORF) 2017 training survey, the annual General Medical Council Training Survey and feedback from trusted colleagues (four trainers and three trainees), two members of the UK-wide ORF Survey Group, the ARENA Clinical Research Fellow in Wales and the Training Programme Director (TPD) for Clinical Oncology training in Northern Ireland. No validated questionnaires have been published to date in this field, most likely due to the lack of enthusiasm for medical education studies in RT learning, and because of the relatively small size of the specialty in each country.
Several questions (and answers) of the questionnaire in this study have been aligned with the ORF's recent national survey ${ }^{34}$ to provide an indication of generalisability. The level of detail in each question item was tailored according to its importance as perceived by those with input to the drafting of this bespoke questionnaire. Response options were carefully tailored for questions on an individual basis, with the use of subjective options in several questions to improve their accessibility for the full range of experience and age within the study population. Important issues raised during phase I that have been omitted from the provisionally approved questionnaire will be added if a supportive consensus is reached by the research team on a question-by-question basis. The research ethics committee will be consulted for approval of the updated questionnaire prior to phase II recruitment.

Questionnaires will be distributed to participants following their confirmation of interest by email response. Paper questionnaires have been chosen over digital formats as the investigators agree that this will yield a better response rate, taking into account the range of levels of seniority among the study population. Completion of questionnaires will take $15 \mathrm{~min}$ and the return of the consent form and questionnaire to a collection box by phase II participants will mark the end of study participation. The collection box will be checked for responses each working day. Results will be recorded with the unique identifier (ranging from 000 to 034) but there is no planned data linkage with phase I or any other datasets. Responses will be transposed to Microsoft Excel and any free-text comments will be recorded in the same spreadsheet.

Descriptive statistics will be applied to summarise the responses and these will be assessed as a pooled group of Clinical Oncology clinicians. The thematic analysis technique will be applied to qualitative questions. This will permit the lead investigator to establish themes among the factors that improved or detracted from the experienced quality of RT learning to date, whether ongoing or previously completed. Coded data resulting from the thematic analysis will allow investigators to organise interview contents in meaningful categories. ${ }^{35}$ Parallels drawn between the described learning environments and the interviewees' accounts of positive or negative aspects may assist in identifying methods to enhance the strengths of modern RT learning and improve any weaknesses identified. The guidance provided for reporting of qualitative survey outcomes will be taken from the SRQR recommendations. ${ }^{36}$

\section{Recruitment}

Recruitment for both phases will begin with an email invitation to all trainers and trainees working at Cancer Centre BCH by hospital email. The email for phase I will describe the study in brief and have the relevant study information and consent form linked as attachments. A date, time and location for an interview will be confirmed 
by email correspondence with the first five trainee and five trainer respondents to register interest. A reserve list of two trainers and trainees will be kept for phase I should some participants have a change in circumstance and two reminder emails will be sent following original invitations, 1 week apart. For phase II, an envelope with the unique 3-digit study number displayed containing the study information, consent form and the questionnaire including instructions will be delivered to the respondents. There will be no limit to the number of participants in phase II. It is expected that recruitment will not be difficult as the study has the support of the region's TPD and Educational Supervisors.

\section{Sample size and statistical analysis}

All specialty trainees $(n=13)$ and consultants $(n=21)$ in Clinical Oncology working at Cancer Centre BCH will be invited to participate in both phases of investigation (investigators excluded). The recruitment target for phase I is based on medical education literature, where a sample of approximately 10 from a homogeneous population is regarded as optimal for conducting IPA. ${ }^{7}$

No statistical tests will be performed on phase I data. Descriptive statistics will be applied to phase II data by the lead investigator. Due to differences between trainers' and trainees' average responses in phase II qualitative data, some will have statistical significance tests applied (decided in advance of survey distribution). Given the small population involved and the uncertainty about accrual, the most suitable statistical test cannot be determined in advance. The power and effect size of such statistics also cannot be predetermined given the small size of the study population. The advice was provided by a senior medical statistician associated with the CME.

\section{Potential sources of bias}

It is possible that study participants may not accurately recall details of their training in years past. This may manifest as proportion of 'not sure' responses or possibly inaccurate responses, especially in the questionnaires among trainers and more senior trainees. It is not possible to reduce this bias; however, study participants will be encouraged to be frank in their responses.

Some Clinical Oncology trainees and trainers may not wish to enrol in phase I as their personal accounts are not blinded to the lead investigator, who is their peer or junior. It will be made clear to phase I study participants that all statements made are made in confidence. Phase II study participants will have their data collected anonymously and therefore their relationship with the lead investigator is irrelevant.

Some of the study participants may be involved in the organisation of the RT training programme or have been involved in the past and could be perceived to have a potential conflict of interest. This connection to the subject matter being studied may affect the responses they offer. Whether such a participant declares involvement will be at their discretion, as no interview stem or questionnaire question addresses this point. Some trainers and trainees may feel that it is their responsibility to defend the RT learning at the Cancer Centre BCH from negative feedback, due to personal attachment to the close-knit community of cancer clinicians working there. Participants are informed in the relevant study information documents that the proposed study seeks to objectively identify both the strengths and weaknesses of the training programme.

Training and service provision matters pertaining to systemic therapy will not be addressed in this study, nor will 'soft skills' acquired during specialty training in preparation for a consultant post, such as leadership and communication.

\section{Patient and public involvement}

No patients were involved.

\section{ETHICS AND DISSEMINATION}

Ethical approval was granted by the School of Medicine, Dentistry and Biological Sciences Research Ethics Committee at QUB (Ref: 17.64) . A successful Integrated Research Application System submission was made through the Belfast Health \& Social Care Trust to secure research governance for research involving employees (Project ID: 242591) . Overall study sponsorship was agreed by QUB (Ref: A18/03) .

The generated results of this mixed-methods study will be shared with the TPD for Clinical Oncology in Northern Ireland, the ORF and the Medical Director for Education and Training at the RCR, so that new findings can be considered in future educational planning both regionally and nationally. The results will also be submitted for publication. Learning points generated from this study may be generalisable and transferrable outside of the UK, given that trainees encounter difficulties in RT education in many countries. ${ }^{24} 37$

To the investigators' knowledge this will be the first research to have been conducted into the learning of RT skills specifically, among trainees who are training in chemotherapy also. It is known that UK trainees will describe challenges in obtaining adequate exposure to RT practice due to competition for their time from outpatient SACT patients and acutely unwell inpatients, ${ }^{3} 2638$ and therefore, a tailored UK study centred on RT training is warranted. This will be the first UK study where both trainers and trainees participate in the same study of RT training. It is reasonable to expect a higher yield of balanced insights from the study by including both stakeholders involved in the learning process. Furthermore, no mixed-methods research of technical RT training is available in the existing literature. The investigators believe that this feature of the study design will lead them to a deeper understanding of the strengths and weaknesses of RT training compared with questionnaires alone and that any importance of the interaction between trainers and trainees may be elucidated. 


\section{Twitter Gerard Walls @gwalls89}

Acknowledgements The investigators wish to thank each of the following individuals for their advice during the development of this study: Miss Mairead Boohan, Dr Caitlin Bowden, Dr Georgina Casswell, Professor Tim Dornan Dr Elin Evans, Dr Jenny Johnston, Mr Mike Stevenson and Professor lan Young. Special thanks must go to those local colleagues who engaged in the design of this study in providing their forthright accounts, and to Miss Andrea Flanagan for providing administrative assistance.

Contributors GW: conception, drafting of study protocol, literature search. JJM and GGH: gave regular review during the iterative refinement process.

Funding The applicant will undertake this research as part of the MSc in Clinical Education at Queen's University Belfast. The tuition fees for the third and final year of the part-time Postgraduate Taught course have been funded by the Dr Gary McGowan Scholarship from Friends of the Cancer Centre. The Scholarship was awarded following competitive application and is offered on an annual basis to an Oncology trainee who is undertaking additional studies during their clinical training. He has subsequently also funded by a Wellcome-HRB Irish Clinical Academic Training Fellowship.

Competing interests None declared.

Patient and public involvement Patients and/or the public were not involved in the design, or conduct, or reporting, or dissemination plans of this research.

Patient consent for publication Not required.

Provenance and peer review Not commissioned; externally peer reviewed.

Open access This is an open access article distributed in accordance with the Creative Commons Attribution Non Commercial (CC BY-NC 4.0) license, which permits others to distribute, remix, adapt, build upon this work non-commercially, and license their derivative works on different terms, provided the original work is properly cited, appropriate credit is given, any changes made indicated, and the use is non-commercial. See: http://creativecommons.org/licenses/by-nc/4.0/.

ORCID iD

Gerard Walls http://orcid.org/0000-0003-0845-9732

\section{REFERENCES}

1 The Royal College of Radiologists. Specialty training curriculum for clinical oncology. London, 2016.

2 Gwynne S, Gilson D, Dickson J, et al. Evaluating target volume delineation in the era of precision radiotherapy: FRCR, revalidation and beyond. Clin Oncol 2017;29:436-8.

3 Kosmin M, Brown S, Hague C, et al. Current views on clinical oncology training from the 2015 oncology registrars' forum survey. Clin Oncol 2016;28:e121-5.

4 The Royal College of Radiologists. Trainee induction pack for specialty training in clinical oncology. London, 2016.

5 The Royal College of Radiologists. Specialty training curriculum for clinical oncology. London, 2007.

6 Choudhury A, Budgell G, MacKay R, et al. The future of imageguided radiotherapy. Clin Oncol 2017;29:662-6.

7 Department of Health. Modernising medical careers the next steps. London, 2005.

8 Council of the European Union. Directive 2000/34/EC of the European Parliament and of the Council of June 2000, amending Council Directive 93/104/EC. Brussels, 2000.

9 NHS Cancer Action Team. Chemotherapy services in England, ensuring quality and safety: a report from the. London: National Chemotherapy Advisory Group, 2009.

10 Black D. The new UK internal medicine curriculum. Clin Med 2017;17:103-4.
11 Benstead K, Gilson D, Hanna L, et al. Training in clinical oncology: results of the Royal College of radiologists' survey of new consultants. Clin Oncol 2012;24:e143-8.

12 October ROM, Society BP, We P, et al. Continuing professional development 2006;19:530-3.

13 NIMDTA. Educational agreement for doctors in training in the Northern Ireland Deanery, 2016.

14 Tailor A, Dubrey S, Das S. Opinions of the ePortfolio and workplacebased assessments: a survey of core medical trainees and their supervisors. Clin Med 2014;14:510-6.

15 Patel P. An evaluation of the current patterns and practices of educational supervision in postgraduate medical education in the UK. Perspect Med Educ 2016;5:205-14.

16 Miller A, Archer J. Impact of workplace based assessment on doctors' education and performance: a systematic review. BMJ 2010;341:c5064.

17 UK Postgraduate Medical Deans. A reference guide for postgraduate specialty training in the UK: the gold guide, 2018.

18 General Medical Council. Standards and guidance: achieving good medical practice. teaching, training, supporting. Manchester, 2016.

19 General Medical Council. Taking revalidation forward: improving the process of relicensing for doctors. Manchester, 2017.

20 Crellin A. Revalidation and lifelong learning. Clin Oncol 2011;23:657-8.

21 The Royal College of Radiologists. Guide to job planning in clinical oncology. Third edition, 2015: 42.

22 The Royal College of Radiologists. Clinical oncology - the future shape of the specialty. London, 2014.

23 Walls GM, Hanna GG, McAleer JJ. Learning radiotherapy: the state of the art. BMC Med Educ 2020;20:150.

24 Debenham B, Banerjee R, Fairchild A, et al. 2009 Canadian radiation oncology resident survey. Int J Radiat Oncol Biol Phys 2012;82:1326-31.

25 Wong DW, Sanghera P, Stevens AM, et al. Survey of West Midlands clinical oncology trainees. Clin Oncol 2008;20:98.

26 Dickson J, Liu D, Bloomfield D. Training in clinical oncology and the transition from trainee to consultant: results of the Royal College of radiologists' 2015 Post-Certificate of completion of training survey. Clin Oncol 2017;29:e64-71

27 Said J, Woolf DK, Glendenning J, et al. The current views of clinical oncology trainees. Clin Oncol 2014;26:159-61.

28 Bibault J-E, Fumagalli I, Diaz O, et al. The French Society of young radiation oncologists: history, goals and perspective. Rep Pract Oncol Radiother 2012;17:255-8.

29 Chapman E, Smith JA. Interpretative phenomenological analysis and the new genetics. $J$ Health Psychol 2002;7:125-30.

30 Giorgi A. Concerning a serious misunderstanding of the essence of the phenomenological method in psychology. J Phenomenol Psychol 2008;39:33-58.

31 King NCassell CSG, ed. Essential quide to qualitative methods in organizational research. London: Sage Publications, 2014: 11-22p.

32 Larkin M, Watts S, Clifton E. Giving voice and making sense in interpretative phenomenological analysis. Qual Res Psychol 2006;3:102-20.

33 Tong A, Sainsbury P, Craig J. Consolidated criteria for reporting qualitative research (COREQ): a 32-item checklist for interviews and focus groups. Int J Qual Health Care 2007;19:349-57.

34 Casswell G, Shakir R, Macnair A, et al. Uk training in clinical oncology: the trainees' viewpoint. Clin Oncol 2018;30:602-4.

35 Fraenkel JR, Wallen NE, Hyun HH. How to design and evaluate research in education. Vol. 53, Climate Change 2013 - The Physical Science Basis. San Francisco, 2013: 436

36 O'Brien BC, Harris IB, Beckman TJ, et al. Standards for reporting qualitative research: a synthesis of recommendations. Acad Med 2014;89:1245-51.

37 Leung J, Rioseco P. Burnout, stress and satisfaction among Australian and New Zealand radiation oncology trainees. $J$ Med Imaging Radiat Oncol 2017;61:146-55.

38 Lei M, Stokoe J, MacLeod N, et al. Clinical oncology training: the trainees' perspective. Clin Oncol 2012;24:22-4. 